\title{
Factorization of Polynomials over Finite Fields
}

\author{
By Robert J. McEliece*
}

\begin{abstract}
If $f(x)$ is a polynomial over $G F(q)$, we observe (as has Berlekamp) that if $h(x)^{q} \equiv h(x)(\bmod f(x))$, then $f(x)=\prod_{a \in G F_{(q)}} \operatorname{gcd}(f(x), h(x)-a)$. The object of this paper is to give an explicit construction of enough such $h$ 's so that the repeated application of this result will succeed in separating all irreducible factors of $f$. The $h$ 's chosen are loosely defined by $h_{i}(x) \equiv x^{i}+x^{i q}+x^{i q}{ }^{2}+\cdots(\bmod f(x))$. A detailed example over $G F(2)$ is given, and a table of the factors of the cyclotomic polynomials $\Phi_{n}(x)(\bmod p)$ for $p=2$, $n \leqq 250 ; p=3, n \leqq 100 ; p=5,7, n \leqq 50$, is included.
\end{abstract}

I. Introduction. The object of this paper is to present a workable algorithm for factoring polynomials over finite fields. The existence of such an algorithm is not in doubt since it is clearly possible to generate recursively all irreducible polynomials of a given degree over a given finite field, and then test any polynomial for divisibility by the irreducibles, one by one; naturally such an algorithm is highly impractical for even low degrees. It is of course frequently necessary to be able to factor polynomials over finite fields; for example in factoring rational primes in algebraic number fields. The algorithm to be given is quite usable; for example over $G F(2)$ it is effective for hand calculations up to degree 15 or so, and with the aid of a computer it is possible to go up to degree several hundred without difficulty. Through the use of this algorithm we have constructed a table, appearing in the microfiche section of this issue, of the factors of $x^{n}-1$ over $G F(p)$ for $p=2$, $n \leqq 250 ; p=3, n \leqq 100 ; p=5, n \leqq 50, p=7, n \leqq 50$. This table gives the factorization of the primes $2,3,5,7$ in the corresponding cyclotomic fields, and is also of use in studying linear recurrence relations of period $n$ over $G F(p)$, since the characteristic polynomials of such recurrences are precisely the divisors of $x^{n}-1$. Published tables of irreducible polynomials over finite fields are insufficient to factor $x^{n}-1$ for even modest values of $n$; for example Marsh's table [1] of polynomials irreducible over $G F(2)$ up to degree 19 cannot be used to factor $x^{43}-1$ over $G F(2)$.

Let us finally mention that Berlekamp [2] has recently published a similar algorithm, which shares Theorem 1, below, with ours, but proceeds in a somewhat different direction. A brief comparison of the two algorithms is given at the end of the next section.

II. The Algorithm. Throughout, let $F=G F(q), q=p^{r}, p$ a prime. If $f(x)$ and $g(x)$ are polynomials over $F$, denote by $(f, g)$ their greatest common divisor, which we assume is monic. (We also adopt the convention $(f, a)=1$ for $a \in F$.) We are given a polynomial $f(x)$ of degree $n$ over $F$, and are asked to write $f$ as a product of irreducible factors. We are free to assume that $f(x)$ is squarefree, since unless $f$ is a

Received February 19, 1968, revised October 3, 1968.

* This paper presents the results of one phase of research carried out at the Jet Propulsion Laboratory, California Institute of Technology, under Contract No. NAS 7-100, sponsored by the National Aeronautics and Space Administration. 
$p$ th power, $f /\left(f, f^{\prime}\right)$ will be a nontrivial squarefree divisor of $f$. And while the algorithm can be applied to an arbitrary polynomial, squarefree or not, to find the irreducible powers which divide it, any preliminary reduction in the degree of $f$ which can be made will shorten the computations. Thus $f(x)$ is henceforth squarefree. We further assume $f(0) \neq 0$. Under these circumstances there will be a smallest integer $e$ such that $f(x) \mid x^{e}-1$ and $(e, p)=1$. $e$ is called the period of $f$.

Theorem 1 gives a way to factor $f$, under certain circumstances:

Theorem 1. If $h(x)^{q} \equiv h(x)(\bmod f(x))$, then

$$
f(x)=\prod_{a \in F}(f(x), h(x)-a) .
$$

Proof. Let $\theta$ be a root of $f$ in a splitting field $K$. Then $h(\theta)^{q}=h(\theta)$ and so $h(\theta)$, being fixed by the Galois group of $K / F$, is an element of $F$. Thus every root of $f$ is a root of exactly one of the polynomials $h(x)-a$, and Theorem 1 follows.

Theorem 1 need not give a nontrivial factorization of $f$; if $h(x) \equiv a(\bmod f(x))$ for some $a \in F$, Theorem 1 is of no use. However, if Theorem 1 does give a nontrivial factorization of $f$, we say that $h$ is an f-reducing polynomial; naturally $h$ is automatically $f$-reducing if $0<\operatorname{deg} h<n=\operatorname{deg} f$. (It will soon develop that $f$-reducing polynomials always exist if $f$ is reducible.) The object of the rest of this section is to indicate a method of constructing $f$-reducing polynomials. There are two possible ways the algorithm could work: first, we could find just one $f$-reducing polynomial, and then inductively proceed to find reducing polynomials for the resulting factors; or, we could produce so many $f$-reducing polynomials that they themselves would reduce all resulting factors of $f$. We shall below give two similar families of $f$-reducing polynomials, corresponding to these two possibilities.

If we discover the least integer $N$ such that $x^{q^{N}} \equiv x(\bmod f(x))$, then $N=1 . c . m$. $\left(n_{1}, n_{2}, \cdots, n_{t}\right)$, where $f(x)=f_{1}(x) f_{2}(x) \cdots f_{t}(x)$ is the factorization of $f$ into irreducibles with $\operatorname{deg} f_{k}=n_{k}$. $N$ is the degree of the splitting field for $f$. Now consider the algebra $R_{f}$ over $G F(q)$ of polynomials $y=y(x)(\bmod f(x))$, and define the map $T(y)=y+y^{q}+y^{q^{2}}+\cdots+y^{q^{N-1}}$. Next we say that $f_{k}$ is a regular divisor of $f$ if $N / n_{k}$ is not divisible by $p$. Note that regular divisors always exist.

THeOREM 2. $T$ is a $G F(q)$-linear function on $R_{f}$ whose rank is equal to the number of regular divisors of $F$. Range $(T) \subseteq G F(q)$ if and only if $f$ is irreducible.

Proof. By a generalization of the well-known Chinese Remainder Theorem [3, p. 63] $R_{f}$ is isomorphic to the direct sum $R_{f_{1}} \oplus \cdots \oplus R_{f_{t}}$ under the map $y \rightarrow$ $\left(y_{1}, y_{2}, \cdots, y_{t}\right)$ with $y \equiv y_{k}\left(\bmod f_{k}(x)\right)$. Since the $f_{k}$ are irreducible, the $R_{f_{k}}$ are fields. Let $T_{k}$ be the trace on $R_{f_{k}}$; i.e., $T_{k}(y)=y+y^{q}+\cdots+y^{q^{n} k^{-1}}$. Then for $y \in R_{f_{k}}, T(y)=m_{k} T_{k}(y)$ where $m_{k}=N / n_{k}$. Thus for $y \in R_{f}, T(y)=$ $T\left(y_{1}, y_{2}, \cdots, y_{t}\right)=\left(m_{1} T_{1}\left(y_{1}\right), \cdots, m_{t} T_{t}\left(y_{t}\right)\right)$, and so if $m_{k}=0$ (i.e., $f_{k}$ is irregular) the $k$ th coordinate of $T(y)$ will be identically zero, and otherwise the $k$ th coordinate ranges freely over $G F(q)$. This shows that dim range $(T)=$ number of regular divisors. To prove the last sentence of Theorem 1 , notice that in the isomorphism $R_{f} \cong R_{f_{1}} \oplus \cdots \oplus R_{f_{t}}, G F(q)$ appears as the diagonal; i.e. $t$-tuples of the form $(a$, $a, \cdots, a), a \in G F(q)$. Clearly if $t \geqq 2$, range $(T)$ cannot be contained in $G F(q)$ since as we have seen the nonzero coordinates of range $(T)$ vary independently from one another. This completes the proof of Theorem 2 .

Now since $1, x, x^{2}, \cdots, x^{n-1}$ are a basis for $R_{f}$ over $G F(q)$, the polynomials 
$T_{i}(x)=T\left(x^{i}\right)=x^{i}+x^{i q}+\cdots+x^{i q N-1}$ span range $(T)$. Furthermore $T_{i}(x)^{q} \equiv$ $T_{i}(x)(\bmod f(x))$, so we arrive at the important

CoRollary 1 . The polynomials $T_{i}(x), 1 \leqq i<n$, include f-reducing polynomials unless $f$ is already irreducible.

Although the polynomials $T_{i}$ of Corollary 1 enable us to begin the factorization of $f$, they are not usually able to reduce all the resulting factors. What is not difficult to show is that the best the $T_{i}$ 's allow is the factorization $f=f_{1} \cdots f_{j} \bar{f}_{j+1}$ where $f_{1}, f_{2}, \cdots, f_{j}$ are the regular divisors of $f$ and $\bar{f}_{j+1}$ is the product of the irregular divisors. Of course what one does in practice is compute the first $f$-reducing $T_{i}$, and then compute new $T_{i}$ for each of the resulting factors. However, it is possible to give another set of polynomials, $R_{i}(x)$, which are capable of separating all the irreducible factors of $f$ at once.

Definition. For each $i, 1 \leqq i<e$, let $m_{i}$ be the least integer such that $x^{i} \equiv$ $x^{i q m_{i}}(\bmod f(x))$. (It is easy to see that $m_{i}=\operatorname{ord}_{e /(e, i)}(q)$, but it is not necessary to know $e$ in order to compute the $m_{i}$.) We define

$$
R_{i}(x) \equiv x^{i}+x^{i q}+\cdots+x^{i q^{m} i^{-1}}(\bmod f(x)) .
$$

Then the $R_{i}$ clearly satisfy $R^{q} \equiv R(\bmod f(x))$, and so they are certainly candidates for $f$-reducing polynomials; indeed $T_{i}(x) \equiv c_{i} R_{i}(x)(\bmod f(x))$ for $c_{i}=N / m_{i}$, so that the $R_{i}$ are certainly no worse than the $T_{i}$. We now show that the $R_{i}, 1 \leqq i<e$, are capable of distinguishing all the factors of $f$. Two easy lemmas are required. We shall see that it is enough to consider the special case $f(x)=x^{e}-1$.

Lemma 1. Let $f(x)=x^{e}-1$ for some e prime to $p$. If $h(x)^{q} \equiv h(x)\left(\bmod x^{e}-1\right)$, then $h(x)$ is a $G F(q)$-linear combination of the polynomials $R_{i}(x)$.

Proof. We first describe the $R_{i}$. According to the definition let $m_{i}$ be the smallest integer such that $x^{i} \equiv x^{i q^{m}}{ }_{i}\left(\bmod x^{e}-1\right)$; i.e., $i \equiv i q^{m_{i}}(\bmod e)$. Hence $R_{i}=x^{i}+$ $x^{i q}+\cdots+x^{i q^{m} i^{-1}}$, and the exponents which occur are precisely the residues $\bmod e$ which are obtained from $i$ by multiplying by various powers of $q$. For example with $q=3, e=13$, the orbits are $(0),(1,3,9),(2,6,5),(4,12,10),(7,8,11)$ and so $R_{1}=R_{3}=R_{9}=x+x^{3}+x^{9} ; R_{2}=R_{6}=R_{5}=x^{2}+x^{5}+x^{6}$, etc. Now suppose $h(x)^{q} \equiv h(x)\left(\bmod x^{e}-1\right)$; if we let $h(x)=\sum_{k=0}^{e-1} h_{k} x^{k}$, then $h(x)^{q} \equiv h\left(x^{q}\right)=$ $\sum h_{k} x^{q k}$, with exponents reduced $\bmod e$, if necessary. Hence $h_{k}=h_{k q}=h_{k q}{ }^{2}=\cdots$ for all $k$, so that $h(x)=\sum_{k} \in_{K} h_{k} R_{k}(x)$, where the set $K$ contains exactly one member from each equivalence class of residues modulo $e$ given by $k_{1} \sim k_{2}$ if and only if $k_{1} \equiv k_{2} q^{t}(\bmod e)$ for some $t \geqq 0$.

Lemma 2. If $f$ is an irreducible divisor of $x^{e}-1$, then there is a polynomial $g$ with $\left(x^{e}-1, f g\right)=f$ and $(f g)^{q} \equiv f g\left(\bmod x^{e}-1\right)$.

Proof. Since $(e, p)=1, x^{e}-1$ is squarefree, and so $\left(f,\left(x^{e}-1\right) / f\right)=1$. Hence there is a $g$ such that $f g \equiv 1\left(\bmod \left(x^{e}-1\right) / f\right)$. This implies $(f g)^{2} \equiv f g\left(\bmod x^{e}-1\right)$ and so also $(f g)^{q} \equiv f g\left(\bmod x^{e}-1\right)$. Finally from $\left(g,\left(x^{e}-1\right) / f\right)=1$ follows $\left(f g, x^{e}-1\right)=f$.

TheOREM 3. Let $f_{1}$ and $f_{2}$ be distinct irreducible divisors of $x^{e}-1$. Then there is an integer $i, 1 \leqq i<e$, and distinct elements $a, b \in F$ such that

$$
R_{i}(x) \equiv a\left(\bmod f_{1}\right), \quad R_{i}(x) \equiv b\left(\bmod f_{2}\right) .
$$

Hence the factors $f_{1}$ and $f_{2}$ can be "separated" by the factorization given in Theorem 1 , using $R_{i}$. 
Proof. Suppose, on the contrary, that for each $i$ there is an element $a_{i} \in F$ such that $R_{i}(x) \equiv a_{i}\left(\bmod f_{1} f_{2}\right)$. By Lemma 2 there exists $h(x)$ such that $\left(f_{1} h\right)^{q} \equiv f_{1} h$ $\left(\bmod x^{e}-1\right)$ and $\left(f_{1} h, x^{e}-1\right)=f_{1}$. Lemma 1 then shows that $f_{1} h \equiv \sum b_{i} R_{i}(x)$ for suitable $b_{i} \in F$. Our assumption implies that $f_{1} h \equiv \sum b_{i} R_{i} \equiv \sum a_{i} b_{i} \equiv$ $b\left(\bmod f_{1} f_{2}\right) ;$ this implies $f_{1} h \equiv b\left(\bmod f_{1}\right)$ so that $b=0$. On the other hand $f_{1} h \equiv 0$ $\left(\bmod f_{1} f_{2}\right)$ is in conflict with $\left(f_{1} h, x^{e}-1\right)=f_{1}$, and the proof is complete.

Corollary. For any squarefree $f(x)$, the corresponding $R_{i}(x), 1 \leqq i<e=$ period $(f)$, will separate all irreducible factors of $f$.

Proof. Denote by $R_{i}^{(e)}(x)$ the $R$ 's associated with $x^{e}-1$. Theorem 3 shows us that the $R_{i}^{(e)}(x)$ suffice to separate all factors of $x^{e}-1$, so they certainly suffice to separate the factors of $f$. On the other hand $x^{i q} \equiv x^{i}\left(\bmod x^{e}-1\right)$ certainly implies that $x^{i q}{ }^{m} \equiv x^{i}(\bmod f(x))$, so that $R_{i}{ }^{(e)}(x) \equiv k_{i} R_{i}(x)(\bmod f(x))$ for suitable $k_{i} \in F$; thus the $R_{i}$ can separate all the factors of $f$. (In fact it is not hard to see that $k_{i}=1$ for all $i$.)

The corollary to Theorem 3 shows that the $R_{i}, 1 \leqq i<e$, will separate the factors of $f$. One might hope, however, that only the $R_{i}, 1 \leqq i<n$, would be needed, but this is not always the case. For example over $G F(2)$, if $f(x)=f_{1} f_{2} f_{3}$ with $\operatorname{deg} f_{1}=$ $\operatorname{deg} f_{2}=4, \operatorname{deg} f_{3}=8$, then $R_{1}, \cdots, R_{11}$ cannot separate $f_{1}$ from $f_{2}$. Hence the disadvantage in using the $R_{i}$ is that it is in general necessary to compute a large number of them in order to be sure they will separate all factors. However, in the important special case $f(x)=x^{e}-1$, the $R_{i}$ are ideally suited. (See Example 2, below.)

Comparison with Berlekamp's Algorithm. The central point of Berlekamp's algorithm is that the equation $h(x)^{q}-h(x) \equiv 0(\bmod f(x))$ may be regarded as a homogeneous system of $n$ simultaneous linear equations in the coefficients of $h$. Thus Berlekamp finds $f$-reducing polynomials by finding the nullspace of a certain $n \times n$ matrix over $G F(q)$. This amounts to row-reducing an $n \times n$ matrix, which turns out to require on the order of $n^{3}$ coordinate operations over $G F(q)$, and the amount of calculation is not highly dependent upon the polynomial being factored.

On the other hand, the analysis of the algorithm of this paper is not so simple, for the amount of calculation required depends very heavily on the integer $N$ which in turn is highly sensitive to the factorization of $f$. For example consider squarefree polynomials $f(x)$ of degree 12 over $G F(2)$; if $f(x)$ is the product of the three irreducibles of degree four, $N=4$, while degrees 3,4 and 5 give $N=60$. The mean value for $N$ among all squarefree polynomials of degree 12 which have no linear factor is 16.4, and it seems reasonable to conjecture that the mean value of $N$ grows linearly with $n$. (But one can show that the largest possible value of $N$ grows faster than $\exp \left(n^{a}\right)$ for all $a<\frac{1}{2}$.) Thus to compute $T_{i}(x)$, one needs $N$ successive $q$ th powers of $x^{i}$ (modulo $f(x)$ ), which requires $n^{2} N$ coordinate operations. And since in general several $T_{i}$ must be computed before an $f$-reducing polynomial is found, this algorithm is no better than Berlekamp's. However, the process of computing successive $q$ th powers modulo $f$ is a less complex operation than row-reducing an $n \times n$ matrix, so that the present algorithm is, for example, easier to program.

\section{Examples.}

1. Let us apply the algorithm to the polynomial $f(x)=x^{17}+x^{14}+x^{13}+x^{12}$ $+x^{11}+x^{10}+x^{9}+x^{8}+x^{7}+x^{5}+x^{4}+x+1$ over $G F(2) . f(0)=1$ and $f$ is not 
a square. Now $f^{\prime}=x^{16}+x^{12}+x^{10}+x^{8}+x^{6}+x^{4}+1$. We compute $\left(f, f^{\prime}\right)$ by Euclid's algorithm, abbreviating a polynomial $\sum_{i=0}^{n} a_{i} x^{i}$ by the $(n+1)$-tuple $\left(a_{n} a_{n-1} \cdots a_{1} a_{0}\right)$ :

$$
\begin{aligned}
& \begin{array}{llllllllllllllllll}
1 & 0 & 0 & 1 & 1 & 1 & 1 & 1 & 1 & 1 & 1 & 0 & 1 & 1 & 0 & 0 & 1 & 1
\end{array} \\
& \begin{array}{rllllllllllllllll}
1 & 0 & 0 & 0 & 1 & 0 & 1 & 0 & 1 & 0 & 1 & 0 & 1 & 0 & 0 & 0 & 1 \\
\hline 1 & 0 & 1 & 0 & 1 & 0 & 1 & 0 & 0 & 0 & 1 & 0 & 0 & 0 & 1
\end{array} \\
& \begin{array}{rllllllllllllllll}
1 & 0 & 0 & 0 & 1 & 0 & 1 & 0 & 1 & 0 & 1 & 0 & 1 & 0 & 0 & 0 & 1 \\
\hline 1 & 0 & 0 & 0 & 0 & 0 & 1 & 0 & 0 & 0 & 1 & 0 & 1 & 0 & 1
\end{array} \\
& \begin{array}{rrrrrrrrrrrrrrr}
1 & 0 & 1 & 0 & 1 & 0 & 1 & 0 & 0 & 0 & 1 & 0 & 0 & 0 & 1 \\
\hline 1 & 0 & 1 & 0 & 0 & 0 & 0 & 0 & 0 & 0 & 1 & 0 & 0
\end{array} \\
& \begin{array}{lllllllllllllll}
1 & 0 & 1 & 0 & 1 & 0 & 1 & 0 & 0 & 0 & 1 & 0 & 0 & 0 & 1 \\
\hline 1 & 0 & 1 & 0 & 0 & 0 & 0 & 0 & 0 & 0 & 1
\end{array} \\
& \begin{array}{llllllllllll}
1 & 0 & 1 & 0 & 0 & 0 & 0 & 0 & 0 & 0 & 1 \\
\hline
\end{array}
\end{aligned}
$$

Hence $\left(f, f^{\prime}\right)=x^{10}+x^{8}+1$, and an easy division gives $f /\left(f, f^{\prime}\right)=x^{7}+x^{5}+x^{4}$ $+x+1=\bar{f}$, which we now know to be squarefree. We now compute the $T_{i}(x)$, and to do so it is convenient to have a list of even powers of $x$ modulo $\bar{f}$ :

$$
\begin{array}{lllllllll}
x^{0}= & 0 & 0 & 0 & 0 & 0 & 0 & 1 \\
x^{2}= & 0 & 0 & 0 & 0 & 1 & 0 & 0 \\
x^{4}= & 0 & 0 & 1 & 0 & 0 & 0 & 0 \\
x^{6}= & 1 & 0 & 0 & 0 & 0 & 0 & 0 \\
x^{8}= & 1 & 1 & 0 & 0 & 1 & 1 & 0 \\
x^{10}= & 1 & 0 & 0 & 1 & 1 & 0 & 1 \\
x^{12}= & 1 & 0 & 1 & 0 & 0 & 1 & 0
\end{array}
$$

(Berlekamp observed that the operation of squaring a polynomial

$$
\sum_{i=0}^{n-1} a_{i} x^{i} \bmod f(x)
$$

is the same as multiplying the vector $a_{0} a_{1} \cdots a_{n-1}$ by the $n \times n$ matrix of even powers.) We compute $T_{1}$ :

$$
\begin{array}{lllllllll}
x & = & 0 & 0 & 0 & 0 & 0 & 1 & 0 \\
x^{2} & = & 0 & 0 & 0 & 0 & 1 & 0 & 0 \\
x^{4} & = & 0 & 0 & 1 & 0 & 0 & 0 & 0 \\
x^{8} & = & 1 & 1 & 0 & 0 & 1 & 1 & 0 \\
x^{16} & = & 0 & 0 & 0 & 1 & 0 & 1 & 1 \\
x^{32} & = & 1 & 0 & 0 & 0 & 1 & 0 & 1 \\
x^{64} & = & 1 & 0 & 0 & 0 & 0 & 1 & 1 \\
x^{128} & = & 1 & 0 & 1 & 0 & 1 & 1 & 1 \\
x^{256} & = & 0 & 1 & 0 & 0 & 0 & 0 & 1 \\
x^{512} & = & 1 & 0 & 0 & 1 & 1 & 0 & 0 \\
T_{1}(x) & = & 1 & 0 & 0 & 0 & 1 & 1 & 1
\end{array}
$$

$x^{2^{10}}=x$, hence $N=10$.

$T_{1}(x)$ is therefore an $\bar{f}$-reducing polynomial, so

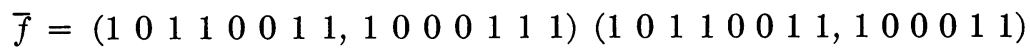

must be a nontrivial factorization: 


$$
\begin{array}{lllllllll}
1 & 0 & 1 & 1 & 0 & 0 & 1 & 1 \\
1 & 0 & 0 & 0 & 1 & 1 & 1 & \\
\hline \\
\hline
\end{array}
$$

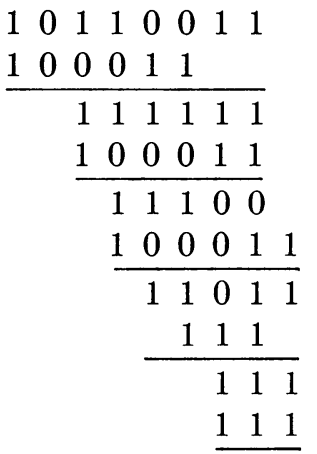

Hence $\bar{f}=\left(\begin{array}{llllll}1 & 1 & 1 & 1 & 0 & 1\end{array}\right)\left(\begin{array}{lll}1 & 1 & 1\end{array}\right)$. Of course 111 is irreducible, and it remains to investigate 111101 . The matrix of even powers modulo 111101 is obtained by reducing the corresponding matrix for $\bar{f}$ by reducing mod 111101 :

$$
\begin{aligned}
& x^{0}=\begin{array}{lllll}
0 & 0 & 0 & 0 & 1
\end{array} \\
& x^{2}=\begin{array}{lllll}
0 & 0 & 1 & 0 & 0
\end{array} \\
& x^{4}=\begin{array}{lllll}
1 & 0 & 0 & 0 & 0
\end{array} \\
& x^{6}=0 \begin{array}{llll}
0 & 1 & 1 & 1
\end{array} \\
& x^{8}=11100 \\
& x=\begin{array}{lllllll}
0 & 0 & 0 & 1 & 0
\end{array} \\
& x^{2}=\begin{array}{lllllll}
0 & 0 & 1 & 0 & 0
\end{array} \\
& x^{3}=\begin{array}{llllll}
0 & 1 & 0 & 0 & 0
\end{array} \\
& x^{4}=\begin{array}{llllll}
1 & 0 & 0 & 0 & 0
\end{array} \\
& x^{6}=\begin{array}{lllllll}
0 & 0 & 1 & 1 & 1
\end{array} \\
& x^{8}=\begin{array}{lllll}
1 & 1 & 1 & 0 & 0
\end{array} \\
& x^{12}=\begin{array}{lllll}
12 & 0 & 1 & 0 & 1
\end{array}
\end{aligned}
$$

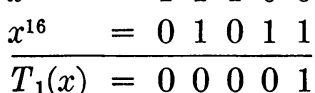

$$
\begin{aligned}
& x^{2^{5}}=x, N=5 \\
& x^{24}=\begin{array}{llllll}
0 & 1 & 1 & 0 & 1
\end{array}
\end{aligned}
$$

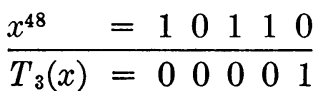

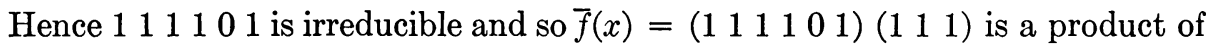
irreducibles. (Actually in this case we could deduce that 111101 was irreducible from $N=10$ for $\bar{f}$, since any factorization of 111101 would have led to a different $N$.) Next we check to see whether or not $\left(f, f^{\prime}\right)$ is divisible by either of the two factors already found. $\left(f, f^{\prime}\right)=\left(\begin{array}{llllll}1 & 1 & 0 & 0 & 0 & 1\end{array}\right)^{2}$, so we need only check for divisibility by 111 , and it is easily found that $1100001=\left(\begin{array}{lll}1 & 1 & 1\end{array}\right)\left(\begin{array}{llll}1 & 0 & 1 & 1\end{array}\right)$. Hence

$$
f(x)=\left(x^{5}+x^{4}+x^{3}+x^{2}+1\right)\left(x^{3}+x+1\right)^{2}\left(x^{2}+x+1\right)^{3}
$$

is the complete factorization.

2. Consider the factorization of the polynomials $x^{e}-1$ over $G F(p), p$ a prime. There is no loss in assuming that $(e, p)=1$, since if $e=e_{1} p^{t}$, then $x^{e}-1=\left(x^{e_{1}}-1\right)^{p^{t}}$. In this special case, the computation of the $R_{i}$ is very simple (see proof of Lemma 1); one need only compute the orbits of the residues mod $e$ under the cyclic permutation group generated by $i \rightarrow i p(\bmod e)$, and these orbits contain the exponents which occur in the various $R_{i}$. For example with $p=3$, $e=20$ the orbits are

$$
\text { (0), }(1,3,9,7)(2,6,18,14)(4,12,16,8)(5,15)(10)(11,13,19,17) \text {, }
$$

and so the corresponding $R_{i}$ are $R_{1}(x)=x+x^{3}+x^{7}+x^{9}, R_{2}(x)=x^{2}+x^{6}+x^{14}$ 
$+x^{18}, R_{3}(x)=x^{4}+x^{8}+x^{12}+x^{16}$, etc. The algorithm of this paper, using the $R_{i}$ 's, was programmed on an SDS-930 computer, and produced the table appearing in the microfiche section of this issue.

Notes on the Table in the Microfiche Section: For a given $e$ only the irreducible factors of $x^{e}-1$ which are not factors of $x^{e^{\prime}}-1$ for $e^{\prime}<e$ are given, so what we have really is a table of the factorization of the cyclotomic polynomials $\Phi_{e}(x)$ of order $e$, $\operatorname{deg} \Phi_{e}(x)=\phi(e)$. The complete factorization is obtained from the formula $x^{e}-1=$ $\prod_{\left.d\right|_{e}} \Phi_{d}(x)$. As is well known, the irreducible factors of $\Phi_{e}(x)$ are all of the same degree $=\operatorname{ord}_{e}(p)$, and in fact the shape of the complete factorization may be seen from the orbits used to calculate the $R_{i}$. In the example given above, the orbit structure shows that $x^{20}-1$ is a product of four irreducibles of degree 4 , one of degree 2 and two of degree one. The orbits $(1,3,9,7)$ and $(11,13,19,17)$ exhaust the residues prime to 20 , so that $\Phi_{20}(x)$ is a product of two irreducibles of degree 4 .

If a polynomial $f(x)=a_{0}+a_{1} x+\cdots+a_{m} x^{m}$ divides $\Phi_{e}(x)$, then so does its reciprocal polynomial $\tilde{f}(x)=a_{m}+a_{m-1} x+\cdots+a_{0} x^{m}$, and only one member of a reciprocal pair is listed. For those $e$ which divide an integer of the form $p^{t}+1$, each irreducible divisor of $\Phi_{e}(x)$ is self-reciprocal; this is indicated by a " $P$ " (since the polynomials are then palindromes) after the entry $e$. When $e$ is either an odd prime $r$ (or twice an odd prime) and $\Phi_{e}(x)=x^{r-1}+x^{r-2}+\cdots+x+1$ (or $x^{r-1}-x^{r-2}+$ $\cdots-x+1)$ is irreducible, the entry " $I$ " is given. Also, for some values of $e=f g$ the irreducible divisors of $\Phi_{e}(x)$ may be obtained from those of period $f$ by replacing $x$ by $x^{g}$. This is indicated by the entry $(f \cdot g)$.

Finally, for $p=2$ and 3 the entries are coded. Binary polynomials are given the customary octal representation; e.g., 7053 represents $x^{11}+x^{10}+x^{9}+x^{5}+x^{3}+$ $x+1$. Ternary polynomials are coded in the base 9 ; e.g., 378 represents $x^{5}+2 x^{3}+$ $x^{2}+2 x+2$. Polynomials for $p=5$ and $p=7$ are not coded; i.e., the coefficients are read directly from the table entries.

Information Processing

Jet Propulsion Laboratory

Pasadena, California 91103

1. R. W. MARsh, Table of Irreducible Polynomials over $G F(2)$ through Degree 19, NSA, U.S. Department of Commerce, Office of Tech. Service, Washington, D.C., 1951.

2. E. R. Berlekamp, "Factoring polynomials over finite fields," Bell System Tech. J., v. 46, 1967, pp. 1853-1859. MR 36 \#2314.

3. S. LA NG, Algebra, Addison-Wesley, Reading, Mass., 1965. MR 33 \#5416. 